\title{
Corrigendum
}

\section{Investigation of MI/M4 Muscarinic Receptors in the Anterior Cingulate Cortex in Schizophrenia, Bipolar Disorder, and Major Depression Disorder}

Katerina Zavitsanou, Andrew Katsifis, Filomena Mattner and Xu-Feng Huang

Neuropsychopharmacology (2006) 31, 896. doi:10.1038/s..npp. I301021

Correction to: Neuropsychopharmacology (2004) 29, 619-625. doi:10.1038/sj.npp.1300367.

Following the publication of this article, the authors noted the author names were listed in an incorrect order. The correct order is shown above. 